\title{
Probing antiprotonic helium with precise lasers
}

\section{Masaki Hori}

The antiproton mass in units of the electron mass is determined with parts-per-billion scale precision by studying an exotic helium atom with two-photon laser spectroscopy.

Antiprotons are the antimatter counterpart of protons, which are assumed to have exactly the same mass and absolute value of charge (but opposite sign) as protons. This is a consequence of a fundamental symmetry of nature called CPT, which stands for charge conjugation, parity, and time reversal. It postulates that if all matter in the universe were replaced with antimatter, if left and right were inverted (meaning vectorial quantities such as velocity or electric field would reverse direction), and if the flow of time were reversed, this 'anti-world' would be indistinguishable from our normal matter world. If we could detect any deviation, however small, it would indicate that this fundamental symmetry is broken. 'Small' is the key word here: it is important to compare matter and antimatter with the highest possible precision. Our aim is to determine the antiproton mass in units of the electron mass, and compare it with the corresponding value for the proton to validate CPT symmetry. The proton-to-electron mass ratio is currently known to a fractional precision of $4 \times 10^{-10}$.

Particle accelerator facilities have produced antiprotons for many years, but they are notoriously difficult to handle because when antiprotons come into contact with ordinary matter, they are absorbed by the atomic nuclei and annihilate within a picosecond. However, nature provides an exception to this rule. Some of the antiprotons stopped in helium gas survive for longer due to the spontaneous formation of an exotic helium atom called antiprotonic helium (chemical symbol $\bar{p} \mathrm{He}^{+} \equiv \bar{p}+\mathrm{He}^{++}+e^{-}$, where $\bar{p}$ is an antiproton, $\mathrm{He}^{++}$is a double-ionized helium atom, and $e^{-}$is an electron). ${ }^{1,2}$ Within this half-matter, half-antimatter atom, an antiproton replaces one of the two electrons that normally orbit around the helium nucleus: see Figure 1. Since this antiproton orbital has a circular shape with a diameter of about $100 \mathrm{pm}$ that has very little overlap with the atomic nucleus, the antiproton can evade annihilation for many microseconds. The remaining electron is retained

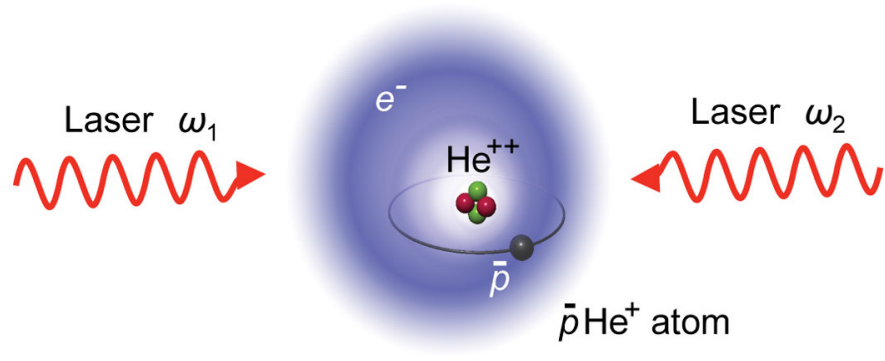

Figure 1. Schematic drawing of an antiprotonic atom $\left(\bar{p} \mathrm{He} e^{+}\right)$excited by two counter-propagating laser beams of optical frequencies $\omega_{1}$ and $\omega_{2} \cdot \bar{p}$ : Antiproton. He ${ }^{++}$: Double-ionized helium atom. $e^{-}$: Electron.

in a spherical, ground-state orbital that protects the antiproton during atomic collisions with other helium particles.

As with other atoms, irradiating a sample of $\bar{p} \mathrm{He}^{+}$with a laser beam precisely tuned to one of its characteristic atomic frequencies can excite resonant transitions between the antiproton orbitals. By comparing the measured frequencies with the results of three-body quantum electrodynamic calculations, ${ }^{3}$ it is possible to determine the antiproton mass relative to the electron mass with high precision. ${ }^{4}$

An important source of uncertainty in our previous experiments arose because of the random thermal motions of $\bar{p} \mathrm{He}^{+}$ atoms in our samples. Atoms moving toward the laser beam experience a different optical frequency compared to those moving away. This Doppler effect broadens the observed spectral lines of $\bar{p} \mathrm{He}^{+}$-see Figure 2(a)—which limited the precision of our previous mass determinations to several parts in $10^{7}-10^{8} .4$

To go beyond this limit, we recently employed a two-photon laser spectroscopy technique. ${ }^{1,5}$ We allowed the $\bar{p} \mathrm{He}^{+}$to simultaneously absorb a photon from each of two counterpropagating laser beams (see Figure 1) so that the red-shift relative to one beam was partially canceled by a corresponding blue-shift in the other. This revealed some narrow spectral lines of $\bar{p} \mathrm{He}^{+}$for the first time: see Figure 2(b).

Continued on next page 
The two-photon transition is difficult to observe because it has a very low probability of occurring within the microsecond-scale lifetime of $\bar{p} \mathrm{He}^{+}$. We efficiently drove it using two high-power, pulsed nanosecond lasers with low spectral noise. ${ }^{6}$ We enhanced the transition probability by many orders of magnitude by carefully tuning the laser frequencies $\omega_{1}$ and $\omega_{2}$ so that the virtual intermediate state of the two-photon transition was within a few gigahertz of a real $\bar{p} \mathrm{He}^{+}$state.

We carried out these experiments at the CERN laboratory in Geneva using the antiproton beams provided by the Antiproton Decelerator facility. The spectrum of Figure 2(a) corresponds to a transition $(n, \ell)=(35,33) \rightarrow(34,32)$ of an isotope of antiprotonic helium $\left(\bar{p}^{4} \mathrm{He}^{+}\right)$measured using a conventional single-photon technique involving a single laser beam. Here the principal and orbital angular momentum quantum numbers of the antiproton orbital are denoted by $n$ and $\ell$. Figure 2 shows the intensity of the spectroscopic signal plotted as a function of the laser frequency. The broad resonance line is caused by the aforementioned Doppler- and power-broadening effects. On the other hand, the two-photon spectrum of Figure 2 (b) corresponds to the transition $(n, \ell)=(36,34) \rightarrow$ $(34,32)$, measured by irradiating two counter-propagating laser beams of wavelengths 372 and $417 \mathrm{~nm}$. Thanks to the higher spectral resolution afforded by this sub-Doppler technique, several $\bar{p} \mathrm{He}^{+}$lines (due to the interactions

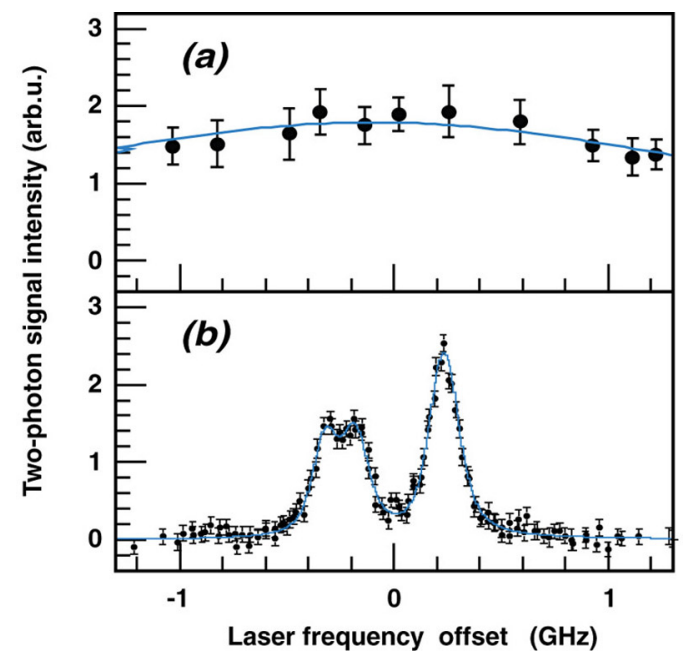

Figure 2. (a) Spectral line of antiprotonic helium measured by singlephoton laser spectroscopy showing a broadened resonance, which is due to the thermal Doppler effect. (b) Two-photon laser spectroscopy reveals a far higher resolution, with multiple atomic lines arising from the spin interactions between the antiproton and electron. Solid lines indicate the best fits of theoretical line profiles. arb.u.: Arbitrary units.

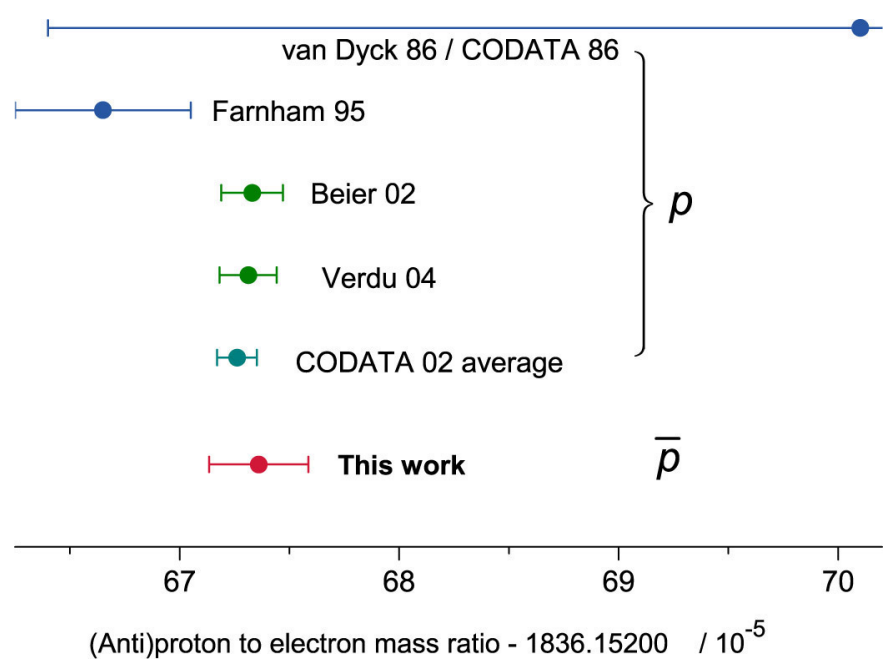

Figure 3. Antiproton-to-electron mass ratio determined by laser spectroscopy of antiprotonic helium ('this work') compared with the protonto-electron mass ratios measured in previous experiments ${ }^{7-9}$ and the Committee on Data for Science and Technology (CODATA) 2002 recommended value obtained by averaging them. ${ }^{10} \mathrm{p}$ : Proton.

between the spins of the antiproton and electron) are now revealed.

The new experiments showed that the antiproton is 1836.1526736(23) times more massive than the electron, the parenthesis indicating the 1-standard deviation error. ${ }^{1}$ This is in good agreement with several previous measurements of the corresponding proton value (see Figure 3). ${ }^{7-9}$ The Committee on Data for Science and Technology (CODATA) ${ }^{10}$ averaged these experimental results to recommend a value of the proton-to-electron mass ratio of $1836.15267245(75)$. The fact that the antiproton-to-electron mass ratio and the proton-to-electron mass ratio agree so well can be interpreted as a verification of the CPT symmetry of nature.

The two-photon technique will allow us to achieve much higher precisions in the future, so that ultimately the antiprotonto-electron mass ratio may be better known than the proton one. We are currently attempting to cool down our $\bar{p} \mathrm{He}^{+}$atoms to low temperatures, which would help us improve the experimental precision of our mass determination.

The author is indebted to the CERN ASACUSA (Atomic Spectroscopy And Collisions Using Slow Antiprotons) collaboration, and to J. Alnis, D. Bakalov, J. Eades, R. Holzwarth, V. I. Korobov, M. Mitani, W. Pirkl, and T. Udem. He thanks the Antiproton Decelerator team for their efforts in providing the antiproton beam. 


\section{Author Information}

\section{Masaki Hori}

Max Planck Institute of Quantum Optics

Garching, Germany

Masaki Hori is a research group leader working on a laser spectroscopy experiment of antiprotonic helium atoms being carried out at CERN's Antiproton Decelerator.

\section{References}

1. M. Hori, A. Sótér, D. Barna, A. Dax, R. Hayano, S. Friedreich, B. Juhász, et al. Two-photon laser spectroscopy of antiprotonic helium and the antiproton-to-electron mass ratio, Nature 475, pp. 484-488, 2011.

2. R. S. Hayano, M. Hori, D. Horváth, and E. Widmann, Antiprotonic helium and CPT invariance, Rep. Prog. Phys. 70, pp. 1995-2065, 2007.

3. V. I. Korobov, Calculations of transitions between metastable states of antiprotonic helium including relativistic and radiative corrections of order $R_{\infty} \alpha^{4}$, Phys. Rev. A 77, p. 042506, 2008.

4. M. Hori, A. Dax, J. Eades, K. Gomikawa, R. S. Hayano, N. Ono, W. Pirkl, et al., Determination of the antiproton-to-electron mass ratio by precision laser spectroscopy of $\bar{p} \mathrm{He}^{+}$, Phys. Rev. Lett. 96, p. 243401, 2006.

5. M. Hori and V. I. Korobov, Calculation of transition probabilities and ac Stark shifts in two-photon laser transitions of antiprotonic helium, Phys. Rev. A 81, p. 062508, 2010. 6. M. Hori and A. Dax, Chirp-corrected, nanosecond Ti:sapphire laser with $6 \mathrm{MHz}$ linewidth for spectroscopy of antiprotonic helium, Opt. Lett. 34, pp. 1273-1275, 2009. 7. D. L. Farnham, R. S. Van Dyck Jr., and P. B. Schwinberg, Determination of the electron's atomic mass and the proton/electron mass ratio via Penning trap mass spectroscopy, Phys. Rev. Lett. 75, pp. 3598-3601, 1995.

8. T. Beier, H. Häffner, N. Hermanspahn, S. G. Karshenboim, H.-J. Kluge, W. Quint, S. Stahl, J. Verdú, and G. Werth, New determination of the electron's mass, Phys. Rev. Lett. 88, p. 011603, 2002.

9. J. Verdú, S. Djekić, S. Stahl, T. Valenzuela, M. Vogel, G. Werth, T. Beier, H.-J. Kluge, and W. Quint, Electronic $g$ factor of hydrogenlike oxygen ${ }^{16} \mathrm{O}^{7+}$, Phys. Rev. Lett. 92, p. 093002, 2004.

10. P. J. Mohr and B. N. Taylor, CODATA recommended values of the fundamental physical constants 2002, Rev. Mod. Phys. 77, pp. 1-107, 2005. 